1 LONG-TERM PROGNOSIS AFTER ACUTE ST-SEGMENT ELEVATION MYOCARDIAL INFARCTION IS DETERMINED BY CHARACTERISTICS IN BOTH NON-INFARCTED AND INFARCTED MYOCARDIUM ON CARDIOVASCULAR MAGNETIC RESONANCE IMAGING

1,2,3Mayooran Shanmuganathan, ${ }^{2}$ Ambra Masi, 2,3 Matthew K Burrage ${ }^{1,3}$ Rafal A Kotronias, ${ }^{1}$ Alessandra Borlotti, ${ }^{1,3}$ Roberto Scarsini, ${ }^{1}$ Abhirup Banerjee, ${ }^{1,3}$ Dimitrios Terentes-Printzios, ${ }^{2}$ Qiang Zhang, ${ }^{2}$ Evan Hann, ${ }^{2}$ Elizabeth Tunnicliffe, ${ }^{3}$ Andrew Lucking, ${ }^{3}$ Jeremy Langrish, ${ }^{3}$ Rajesh Kharbanda, ${ }^{1,3}$ Giovanni Luigi de Maria, ${ }^{1,3}$ Adrian P Banning, ${ }^{1,3}$ Robin P Choudhury, ${ }^{1,3}$ Keith M Channon, ${ }^{2}$ Stefan K Piechnik, 1,2,3Vanessa M Ferreira, on behalf of Oxford Acute Myocardial Infarction (OxAMI) Study investigators. 'Acute Vascular Imaging Centre (AVIC), University of Oxford, John Radcliffe Hospital, Oxford, OX3 9DU, UK; ${ }^{2}$ Oxford Centre for Clinical Magnetic Resonance Research (OCMR), John Radcliffe Hospital, NIHR Oxford Biomedical Research Centre, Oxford BHF Centre of Research Excellence, University of Oxford, Oxford, UK; ${ }^{3}$ Oxford University Hospitals NHS Trust, John Radcliffe Hospital, Oxford, OX3 9DU, UK

\subsection{6/heartjnl-2021-BSCMR.1}

Background After acute ST-segment elevation myocardial infarction (STEMI), CMR infarct characteristics including infarct size (IS), microvascular obstruction (MVO) and intramyocardial haemorrhage (IMH) as well as left ventricular ejection fraction (LVEF) carry prognostic value. However, the long-term prognostic value of changes in the non-infarcted myocardium is unknown.

Aim To evaluate acute changes in both the non-infarcted and infarcted myocardium post-STEMI and their long-term predictive value of major adverse cardiac events (MACE) using conventional CMR and T1-mapping indices.

Methods 219 patients undergoing primary percutaneous coronary intervention post-STEMI prospectively underwent CMR (3T) at 2 days and 6 months, with long-term follow-up for MACE - a composite of cardiac death, sustained ventricular arrhythmia and new-onset heart failure. CMR assessed LVEF, IMH, area-at-risk (AAR), IS and MVO using cine, T2weighted, T2*, T1-mapping and late gadolinium enhancement (LGE) imaging. Area without LGE was defined as noninfarcted myocardium (figure 1A). Infarct and non-infarct T1 were derived from T1-maps using anatomically matching LGE images. "High non-infarct T1" was defined as T1>1250 ms $(>2$ SD above normal range $1184 \pm 30 \mathrm{~ms}$ ). Within the infarction, presence of $\mathrm{MVO} / \mathrm{IMH}$ can lower infarct T1; this was dichotomised into "Higher infarct T1" $(\geq 1300 \mathrm{~ms})$ and "Lower infarct T1" (<1300 ms). Conventional CMR markers (LVEF, AAR, IS, MVO, IMH) and novel T1-mapping biomarkers were assessed for their ability to predict MACE using Kaplan-Meir and Cox-regression survival analysis.

Results 22/219 patients experienced a MACE at a median of 4 years (IQR 2.5-6 yrs). High non-infarct T1 was associated with lower LVEF (51 vs $55 \%, \mathrm{p}=0.002$ ) and higher NT-proBNP levels (290 vs $170 \mathrm{pg} / \mathrm{ml}, \mathrm{p}=0.008)$ at 6 months, and a 2.5-fold increased risk of long-term MACE (figure $1 \mathrm{~B}$; $\mathrm{p}=0.035)$. Lower infarct T1, implying $\mathrm{MVO} / \mathrm{IMH}$, was associated with a 3 -fold risk of MACE $(p=0.020)$. AAR and IMH were not significant predictors of MACE. Both non-infarct T1 $(p<0.001)$ and infarct T1 $(p=0.003)$ remained independent predictors of MACE after adjusting for age, history of MI, ischaemic time, and peak troponin; both significantly improved risk-prediction beyond $\mathrm{LVEF}<40 \%$, IS and $\mathrm{MVO}$ (figure C; C-statistic $0.69 \pm 0.06$ vs $0.77 \pm 0.06$, net reclassification index 42\%; $\mathrm{p}=0.027)$.

Conclusions Both acute non-infarct and infarct myocardial T1 on CMR post-STEMI are independent and incremental predictors of long-term MACE beyond conventional CMR biomarkers.

\section{ASSESSING SPLENIC SWITCH-OFF IN ADENOSINE STRESS CMR FOR PATIENTS WITH ATRIAL FIBRILLATION: A PROPENSITY MATCHED STUDY}

Adam Barrishi, John Graby, Ali Khavandi, Amardeep Dastidar, Jonathan Rodrigues. Department of Radiology, Royal United Hospital; Faculty of Health Sciences, University of Bristol; Department of Cardiology, Royal United Hospital; Department for Health, University of Bath; Department of Cardiology, North Bristol NHS Trust

\subsection{6/heartjnl-2021-BSCMR.2}

Background Stress cardiac magnetic resonance imaging (CMR) is a valuable investigation in the functional assessment of coronary artery disease. The phenomenon of splenic switch-off is a validated indicator of adequate vasodilator stress unique to adenosine vasodilator stress CMR. Patients in atrial fibrillation (AF) may have a reduced adenosine response due to a lower
A

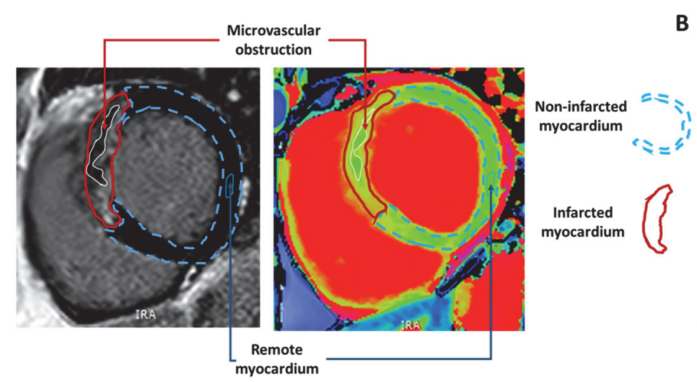

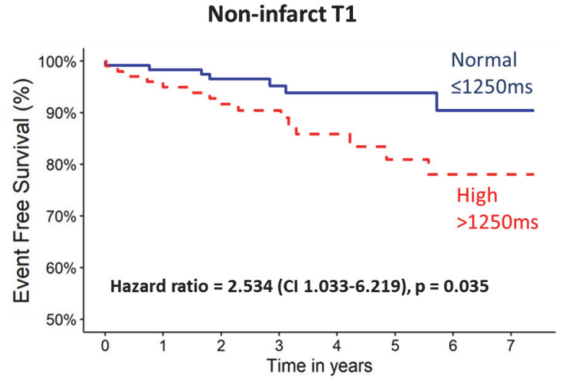

\begin{tabular}{|c|c|c|c|c|c|c|c|c|c|}
\hline \multirow[t]{2}{*}{ Model } & \multirow[t]{2}{*}{ CMR indices } & \multirow[t]{2}{*}{ C-statistic } & \multirow[t]{2}{*}{$\begin{array}{l}\text { Integrated } \\
\text { Brier score }\end{array}$} & \multicolumn{2}{|c|}{ Comparison of models } & \multicolumn{2}{|c|}{ Net Reclassification Index NRI) } & \multicolumn{2}{|c|}{$\begin{array}{l}\text { Integrated discrimination Improvement } \\
\text { Index (IDI) }\end{array}$} \\
\hline & & & & $x^{2}$ difference & $\mathrm{p}$ & index & $\mathrm{p}$ & index & $\mathrm{p}$ \\
\hline $\begin{array}{l}\text { Conventional } \\
\text { CMR indices }\end{array}$ & $\begin{array}{l}\text { Acute LVEF <40\% } \\
\text { MVO presence } \\
\text { Infarct size (per 1\%) }\end{array}$ & $0.69 \pm 0.06$ & 0.075 & \multicolumn{6}{|c|}{$N / A$} \\
\hline \multirow[b]{2}{*}{$\mathrm{T} 1$ indices added } & Acute LVEF $<40 \%$ & \multirow[b]{2}{*}{$0.77 \pm 0.06$} & \multirow[b]{2}{*}{0.064} & \multicolumn{6}{|c|}{ Conventional $\mathrm{CMR}$ model vs model with $\mathrm{T} 1$ indices added } \\
\hline & $\begin{array}{l}\text { Infarct size (per \%) } \\
\text { Infarct T1 (per 1ms) } \\
\text { Non-infarct T1 ( per 1ms) }\end{array}$ & & & 13.21 & 0.001 & $42.3 \%(\mathrm{Cl} 1.4-66.2 \%)$ & 0.027 & $16.1 \%$ (Cl 4.7-30.1\%) & $<0.001$ \\
\hline
\end{tabular}

Abstract 1 Figure 1 (A) T1-maps were segmented into infarcted and non-infarcted myocardium using anatomically matched LGE images. (B) Kaplan-Meier survival analysis of patients with and without high non-infarct T1 on the acute CMR scan after STEMI. (C) Multi-variable Cox regression analysis and comparisons of predictive power of models of conventional and novel CMR indices for predicting long-term MACE were performed. 